\title{
Ácido ascórbico e pigmentos fotossintéticos na alface crespa cultivada em sistema hidropônico com soluções salinas
}

\author{
Ascorbic acid and photosynthetic pigments in curly lettuce grown in a hydroponic system with \\ saline solutions \\ Ácido ascórbico y pigmentos fotosintéticos en lechuga crespa cultivada en sistema hidropónico con \\ soluciones salinas
}

\author{
Josilda de França Xavier \\ ORCID: https://orcid.org/0000-0003-2624-4989 \\ Universidade Federal de Campina Grande, Brasil \\ E-mail: josildaxavier@yahoo.com.br \\ Carlos Alberto Vieira de Azevedo \\ ORCID: https://orcid.org/0000-0001-7336-1243 \\ Universidade Federal de Campina Grande, Brasil \\ E-mail: cazevedo@deag.ufcg.edu.br \\ Márcia Rejane de Q. Almeida Azevedo \\ ORCID: https://orcid.org/0000-0002-4391-2961 \\ Universidade Estadual da Paraíba, Brasil \\ E-mail: mazevedo@ccaa.uepb.edu.br \\ Julio Cesar Rodrigues de Sales \\ ORCID: https://orcid.org/0000-0003-0855-019X \\ Universidade Federal de Campina Grande, Brasil \\ E-mail: julioo5rodrigues@outlook.com \\ Josely Dantas Fernandes \\ ORCID: https://orcid.org/0000-0002-5563-6327 \\ Universidade Estadual da Paraíba, Brasil \\ E-mail: joselysolo@yahoo.com.br \\ Elida Barbosa Corrêa \\ ORCID: https://orcid.org/0000-0003-4660-2202 \\ Universidade Estadual da Paraíba, Brasil \\ E-mail: elida.uepb@gmail.com
}

\begin{abstract}
Resumo
O objetivo desta pesquisa foi determinar os teores de clorofila e vitamina-C nas cultivares da alface (Elba, Cristina e Veneranda) cultivadas em sistema hidropônico com soluções nutritivas apresentando diferentes níveis de salinidade. O experimento foi conduzido na Universidade Estadual da Paraíba,UEPB Campus-II, zona rural da cidade de Lagoa Seca-PB, via técnica Fluxo Laminar de Nutrientes-NFT em ambiente protegido. O delineamento experimental foi em blocos casualizados, em esquema de parcelas subdivididas, as parcelas constituídas por sete soluções nutritivas preparadas a partir da metodologia de Furlani de modo que a solução $S_{1}$ apresentou Condutividade Elétrica-CE (1,9 $\mathrm{dS} \mathrm{m} \mathrm{m}^{-1}$ ) e as demais soluções os seguintes valores de CE: $\mathrm{S}_{2}-2,4 ; \mathrm{S}_{3}-2,9 ; \mathrm{S}_{4}-3,4 ; \mathrm{S} 5-3,9 ; \mathrm{S}_{6}-4,4$ e $\mathrm{S}_{7}-4,9 \mathrm{dS} \mathrm{m}^{-1}, \mathrm{com}$ três repetições. As subparcelas foram compostas pelas cultivares Elba, Cristina e Veneranda. As variáveis analisadas foram: teores de clorofila ( $C h l a r e b)$ total, carotenoides e vitamina-C. As soluções nutritivas apresentaram comportamento linear para todos os tratamentos não deferindo estaticamente entre si. O maior valor para clorofila total foi obtido na cultivar Veneranda. Para as variáveis (Chl b e total) os maiores teores encontrados foi na cultivar Veneranda, cujos valores foram de 35,97 e $52,06 \mathrm{mg} / \mathrm{g}^{-1}$ respectivamente. O tratamento Elba/ $\mathrm{S}_{3}=3,4 \mathrm{dS} \mathrm{m}^{-1}$ proporcionou o maior teor de carotenoides média de $57 \mathrm{mg} / \mathrm{g}^{-1}$, ainda a cultivar Elba apresentou os maiores teores ( Chl a) com as soluções nutritivas $\mathrm{S}_{2}=2,4$ e $\mathrm{S}_{3}=3,9 \mathrm{dS} \mathrm{m} \mathrm{m}^{-1}$. A cultivar Veneranda promoveu os maiores teores para (Chl b e total). Os maiores teores de vitamina-C foram encontrados nas cultivares Cristina e Veneranda.

Palavras-chave: Lactuca sativa L.; Hidroponia; Salinidade; SPAD-502; Clorofila.
\end{abstract}

\begin{abstract}
The objective of this research was to determine the levels of chlorophyll by and vitamin-C in the cultivars of lettuce (Elba, Cristina and Veneranda) in the hydroponic system with nutrient solutions with different levels of salinity. The experiment was conducted at the State University of Paraíba,UEPB Campus-II, rural area of the city of Lagoa SecaPB, using the Laminar Flow of Nutrients-NFT technique in a protected environment. The experimental design was in
\end{abstract}


randomized blocks, in a subdivided plot scheme, the plots consisting of seven nutrient solutions prepared from the Furlani methodology so that solution $\mathrm{S}_{1}$ presented Electric conductivity-EC $\left(1.9 \mathrm{dS} \mathrm{m}^{-1}\right)$ and the other solutions, EC values: $\mathrm{S}_{2}-2.4 ; \mathrm{S}_{3}-2.9 ; \mathrm{S}_{4}-3.4 ; \mathrm{S}_{5}-3.9 ; \mathrm{S}_{6}-4.4$ and $\mathrm{S}_{7}-4,9 \mathrm{dS} \mathrm{m} \mathrm{m}^{-1}$, with three repetitions. The subplots composed of the cultivars Elba, Cristina and Veneranda. Variables analyzed: levels of total chlorophyll ( Chl $a$ and $b$ ), carotenoids and vitamin-C. The nutritional solutions showed linear behavior for all treatments, not differing statically from each other. The highest value for total chlorophyll was obtained in the cultivar Veneranda. For the variables ( $C h l b$ and total) the highest levels were found in the cultivar Veneranda whose values 35.97 and $52.06 \mathrm{mg} \mathrm{g}^{-1}$ respectively. The treatment Elba/ $\mathrm{S}_{3}=3.4 \mathrm{dS} \mathrm{m}{ }^{-1}$ provided the highest average carotenoid content of $57 \mathrm{mg} \mathrm{g}^{-1}$, yet the cultivar Elba showed the highest levels $(C h l a)$ with nutrient solutions $\mathrm{S}_{2}=2.4$ and $\mathrm{S}_{3}=3.9 \mathrm{dS} \mathrm{m}^{-1}$. The cultivar Veneranda promoted the highest levels for ( $C h l b$ and total). The highest levels of vitamin-C were found in the cultivars Cristina and Veneranda.

Keywords: Lactuca sativa L.; Hydroponics; Salinity; SPAD-502; Chlorophyll.

\section{Resumen}

El objetivo de esta investigación fue determinar los niveles de clorofila y vitamina-C en cultivares da lechuga (Elba, Cristina y Veneranda) cultivados en sistema hidropónico con soluciones nutritivas con diferentes niveles de salinidad. El experimento se realizó en la Universidad Estatal de Paraíba,UEPB Campus-II, área rural de la ciudad de Lagoa Seca-PB, la técnica de Flujo Laminar de Nutrientes-NFT en ambiente protegido. El diseño experimental en bloques aleatorios, esquema de parcelas subdivididas, las parcelas consistieron soluciones nutritivas preparadas a partir la metodología Furlani, de manera que la solución $\mathrm{S}_{1}$ presentó Conductividad eléctrica-CE $\left(1.9 \mathrm{dS} \mathrm{m}^{-1}\right)$ y las otras soluciones con CE: $\mathrm{S}_{2}-2.4 ; \mathrm{S}_{3}-2.9 ; \mathrm{S}_{4}-3.4 ; \mathrm{S}_{5}-3.9 ; \mathrm{S}_{6}-4.4$ y $\mathrm{S}_{7}-4.9 \mathrm{dS} \mathrm{m}{ }^{-1}$, con tres repeticiones, las subparcelas compuestas los cultivares Elba, Cristina y Veneranda. Variables analizadas fueron: niveles de clorofila total, Chl a, b, carotenoides y vitamina-C. Las soluciones nutricionales mostraron un comportamiento lineal para todos los tratamientos, no difiriendo estáticamente entre sí. El mayor valor de clorofila total se obtuvo en el cultivar Veneranda. Para las variables (Chl b y total) los niveles más altos se encontraron en el cultivar Veneranda, cuyos valores fueron 35.97 y $52.06 \mathrm{mg} / \mathrm{g}^{-1}$ respectivamente. El tratamiento Elba/ $\mathrm{S}_{3}=3.4 \mathrm{dS} \mathrm{m}{ }^{-1}$ proporcionó el contenido promedio de carotenoides más alto de $57 \mathrm{mg} / \mathrm{g}^{-1}$, sin embargo, el cultivar Elba mostró los niveles más altos (Chl a) con soluciones nutritivas $\mathrm{S}_{2}=2.4$ y $\mathrm{S}_{3}=3.9 \mathrm{dS} \mathrm{m}^{-1}$. El cultivar Veneranda promovió los niveles más altos para (Chl b y total). Los niveles más altos de vitamina-C se encontraron en los cultivares Cristina y Veneranda.

Palabras clave: Lactuca sativa L.; Hidroponia; Salinidad; SPAD-502; Clorofila.

\section{Introdução}

A clorofila pode participar na transferência de energia, durante a qual uma molécula excitada de clorofila transfere sua energia para outra molécula (Taiz et al., 2017). Um dos componentes vegetais mais importantes são os pigmentos, que são compostos orgânicos capazes de absorver a radiação eletromagnética na faixa de 400 a $700 \mathrm{~nm}$ (Kluge et al., 2015).

Os pigmentos fotossintéticos presentes nos vegetais variam de acordo com a espécie. A clorofila $a$ (Chl $a)$ está presente em todos os organismos que realizam fotossíntese exigênica. As bactérias fotossintetizantes são desprovidas de Chl $a$ e possuem, em seu lugar, a bacterioclorofila como pigmento fotossintético. A Chl a é o pigmento utilizado para realizar a fotoquímica, enquanto os demais pigmentos auxiliam na absorção de luz e na transferência da energia radiante para os centros de reação, motivo por que são chamados de pigmentos acessórios (Taiz \& Zieger, 2013).

A Vitamina C possui ação antioxidante onde estes compostos químicos podem prevenir ou diminuir os danos oxidativos de lipídios, proteínas e ácidos nucleicos causados por espécies de oxigênio reativo, que incluem os radicais livres, ou seja, os antioxidantes possuem a capacidade de reagir com os radicais livres e assim restringir os efeitos maléficos ao organismo (Couto \& Canniatti-Brazaca, 2010).

A salinidade é um problema sério para a agricultura, pois limita o crescimento e o desenvolvimento das plantas sensíveis. Nos últimos anos várias pesquisas têm demonstrado o potencial de uso de águas salinas na produção de hortaliças em cultivo hidropônico (Santos et al., 2012).

A alface (Lactuca sativa L.) é uma planta herbácea, originária de clima temperado, pertencente à família Asteracea e da subfamília Cichoriaceae, uma das hortaliças mais populares e consumidas no mundo e no Brasil. A alface se destaca no cenário nacional de cultivos em hidroponia, sendo responsável por aproximadamente $80 \%$ da produção agrícola brasileira desse sistema (Alves et al., 2011). 
O uso da hidroponia surgiu como uma alternativa a problemas como a baixa disponibilidade de solos aptos à agricultura, o interesse em incrementar a eficiência do uso da água e o desejo de aumentar a produção e melhorar a qualidade dos alimentos (Souza Neta et al., 2013).

A qualidade da água utilizada no preparo da solução nutritiva é fator fundamental para se obter elevada produtividade e qualidade dos produtos, seja no cultivo utilizando o sistema NFT (Gondim et al., 2010; Paulus et al., 2010) ou no cultivo em substrato (Dias et al., 2011; Santos et al., 2012). A solução nutritiva para o cultivo hidropônico é de fundamental importância, pois o crescimento e desenvolvimento da cultura dependerão de uma formulação adequada (Oliveira et al., 2014). As oscilações na condutividade elétrica (CE) estão relacionadas ao consumo de água e nutrientes pelas plantas e a evaporação das soluções nutritivas ocorridas durante a condução do experimento (Monteiro Filho et al., 2017). Silva et al. (2015) estudaram dois níveis de disponibilidade de solução nutritiva (2,75 e 5,50 L por maço de 24 plantas) e quatro níveis de condutividade elétrica da água (CE): 0,43 (testemunha); 3,09; 6,87 e 8,53 dS m-1.

O objetivo desta pesquisa foi determinar os teores de clorofila e vitamina $\mathrm{C}$ nas cultivares da alface crespa (Elba, Cristina e Veneranda) cultivadas em sistema hidropônico com soluções nutritivas apresentando diferentes níveis de salinidade.

\section{Metodologia}

O experimento foi conduzido no Centro de Ciências Agrárias e Ambientais-CCAA da Universidade Estadual da Paraíba-UEPB, situado na zona rural da cidade de Lagoa Seca-PB, com as seguintes coordenadas geográficas: $\left(7^{\circ} 10^{\prime} 15^{\prime \prime} \mathrm{S}\right.$, $35^{\circ} 51^{\prime} 14^{\prime \prime} \mathrm{W}$ ), segundo a classificação climática de Köppen-Geige (Brasil, 1971) o clima do município é caracterizado como tropical úmido, com temperatura média anual em torno de $22^{\circ} \mathrm{C}$, sendo a mínima de $18^{\circ} \mathrm{C}$ e a máxima de $33^{\circ} \mathrm{C}$. $\mathrm{O}$ sistema hidropônico utilizado foi o Fluxo Laminar de Nutrientes-NFT instalado em casa de vegetação. Durante a condução do experimento, registraram-se diariamente os valores da temperatura máxima, mínima, média. As temperaturas foram registradas as 8:00h, cujo os dados estão demonstrados na Figura 1.

Figura 1. Valores das temperaturas máxima, mínima, média e T. momento registradas as 8:00h durante a condução do experimento.

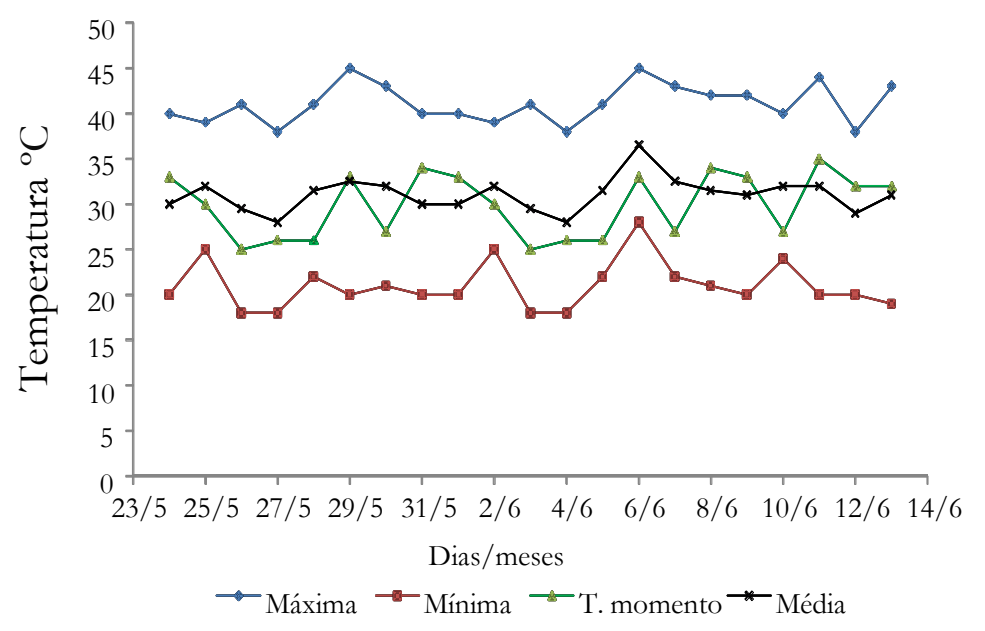

Fonte: Dados da presente pesquisa.

O delineamento do experimento foi em blocos casualizados, em esquema de parcelas subdivididas, sendo as parcelas constituídas por sete soluções nutritivas todas preparadas a partir da metodologia de Furlani (1995) de modo que a solução $\mathrm{S}_{1}$ 
apresentou uma $C E$ de $1,9 \mathrm{dS} \mathrm{m}{ }^{-1}$ e as soluções $S_{2}, S_{3}, S_{4}, S_{5}, S_{6}$ e $S_{7}$ os seguintes valores de CE: 2,4; 2,9; 3,4; 3,9; 4,4 e $4,9 \mathrm{dS}$ $\mathrm{m}^{-1}$, respectivamente. Com exceção da solução $\mathrm{S}_{1}$, as demais soluções tiveram seus valores de CE aumentados pelo acréscimo do Cloreto de sódio $(\mathrm{NaCl})$. Todas as soluções nutritivas foram preparadas com água de chuva com $\mathrm{CE} 0,156 \mathrm{dS} \mathrm{m}^{-1}$ de modo a se obter um volume de 200 litros (L) de solução estoque. Os quantitativos dos fertilizantes minerais e da composição química da solução nutritiva mineral estão dispostos nas Tabelas 1 e 2 .

Tabela 1. Quantitativo dos fertilizantes utilizados na confecção das soluções nutritivas minerais.

\begin{tabular}{|c|c|}
\hline \multicolumn{2}{|l|}{ Solução } \\
\hline Sais & Furlani \\
\hline \multicolumn{2}{|l|}{ g $1000 \mathrm{~L}^{-1}$ de água } \\
\hline $\mathrm{Ca}\left(\mathrm{NO}_{3}\right)_{2} ; 6 \mathrm{H}_{2} \mathrm{O}$ - Nitrato de cálcio & 1000 \\
\hline MAP - Fosfato monoamônio & 150 \\
\hline DAP - Fosfato diamônio & - \\
\hline $\mathrm{H}_{2} \mathrm{PO}_{4}$ - Ácido fosfórico & - \\
\hline $\mathrm{KH}_{2} \mathrm{PO}_{4}$ - Fosfato monopotássico & - \\
\hline $\mathrm{KCl}$ - Cloreto de potássio & 150 \\
\hline KNO3 - Nitrato de potássio & 600 \\
\hline $\mathrm{MgSO}_{4} .7 \mathrm{H}_{2} \mathrm{O}$ - Sulfato de magnésio & 250 \\
\hline MnCl. $\mathrm{H}_{2}$ - O Cloreto de manganês & 2,34 \\
\hline $\mathrm{Mn} \mathrm{SO}_{4} \cdot \mathrm{H}_{2} \mathrm{O}$ - Sulfato de manganês & - \\
\hline $\mathrm{ZnSO}_{4} .7 \mathrm{H}_{2} \mathrm{O}$ - Sulfato de zinco & 0,88 \\
\hline $\mathrm{CuSO}_{4} .7 \mathrm{H}_{2} \mathrm{O}$ - Sulfato de cobre & 0,2 \\
\hline $\mathrm{H}_{3} \mathrm{BO}_{3}$ - Ácido bórico & 2,04 \\
\hline $\mathrm{Na}_{2} \mathrm{MoO}_{4} \cdot 2 \mathrm{H}_{2} \mathrm{O}$ - Molibidato de amônio & 0,26 \\
\hline Fe-EDTA - Ferro EDTa. & $1000 \mathrm{~mL}$ \\
\hline
\end{tabular}

Fonte: Furlani (1995). 
Tabela 2. Composição química das soluções nutritivas minerais.

\begin{tabular}{ccc}
\hline & Solução & \\
\hline Sais & g 1000 L $\mathbf{~}^{-1}$ de água & \\
\hline $\mathrm{NO}_{3}$ - Nitrato & 200,44 \\
$\mathrm{NH}_{4}$ - Amônio & 16,51432 \\
$\mathrm{P}$ - Fósforo & 32,70 \\
$\mathrm{~K}$ - Potássio & 310,275 \\
$\mathrm{Ca}$ - Cálcio & 168 \\
$\mathrm{Mg}$ - Magnésio & 24,65 \\
$\mathrm{~S}$ - Enxofre & 32,50 \\
$\mathrm{Mn}$ - Manganês & 0,636714 \\
$\mathrm{Zn}$ - Zinco & 0,199144 \\
$\mathrm{Cu}$ - Cobre & 0,0671 \\
Bo - Boro & 0,356592 \\
Mo - Molibdênio & 0,114452 \\
Fe- Ferro & 2,234 \\
\hline
\end{tabular}

Fonte: Furlani (1995).

O volume da solução que alimentava o sistema (NFT) durante o cultivo da alface foi acondicionado em baldes plásticos com capacidade para $20 \mathrm{~L}$, entretanto, para se evitar o desperdício de solução por transbordamento do balde, utilizouse o volume de $17 \mathrm{~L}$. Com o intuito de se manter a solução nutritiva dentro dos limites de temperatura recomendados para o cultivo hidropônico, os baldes contendo a solução foram revestidos com folhas de Etileno Acetato de Vinila (E.V.A) com espessura de 5mm, pelo volume registrado após 24:00 h.

A quantificação do volume evapotranspirado pelas plantas (VETc), em função das soluções nutritivas, foi efetuada diariamente, completando o volume do reservatório até o nível de 17 litros, utilizando uma proveta graduada em mililitros conforme a Equação 1.

A VETc foi considerada como uma variável quantitativa para estabelecer uma análise de regressão polinomial.

$$
\begin{aligned}
& \text { VETc }=\frac{V i}{n . \Delta t} \\
& V E T c-\text { volume evapotranspirado, em L planta- }{ }^{-1} \mathrm{dia}^{-1} ; \\
& V i-\text { volume de solução consumida do intervalo de tempo de } 24 \mathrm{~h} ; \\
& \Delta T-\text { intervalo de tempo entre as leituras, dias; } \\
& n-\text { numero de plantas no perfil no intervalo de tempo, } \Delta \mathrm{T} .
\end{aligned}
$$

Durante a condução do experimento o monitoramento ocorreu diariamente, em intervalos de 24:00, realizando leituras das soluções e a calibração do potencial hidrogeniônico $(\mathrm{pH})$, da condutividade elétrica $(\mathrm{CE})$, utilizando-se equipamento portátil para a leitura de pH, CE e sólidos totais dissolvidos (TDS)/Temperatura modelo HI 9811-5. Após a calibração, o pH das soluções foram ajustados, mantendo-os próximos à neutralidade, com a utilização de uma solução de hidróxido de sódio $(\mathrm{NaOH})$ ou Ácido clorídrico (HCL) $\left(1 \mathrm{~mol} \mathrm{~L}^{-1}\right)$. A variação do pH foi feita considerando a média da concentração molar dos íons de hidrogênio $(\mathrm{H}+)$ utilizando a expressão $\mathrm{pH}=-\log [\mathrm{H}+]$. 
Os níveis da CE de todas as soluções foram mantidos levando em consideração $20 \%$ para mais ou $20 \%$ para menos da CE inicial. Quando a CE em cada solução aparentava 20\% maior da inicial a reposição era realizada com água de chuva. Quando a CE apresentava 20\% menor da inicial a reposição era feita com a solução estoque de cada solução nutritiva. O manejo das soluções nutritivas foi realizados diariamente através da reposição da água consumida, do acompanhamento da CE e pH mantendo-o próximo à neutralidade, com a utilização de uma solução de $\mathrm{NaOH}$ ou HCL $\left(1 \mathrm{~mol} \mathrm{~L}^{-1}\right)$ e independente dos tratamentos, as soluções nutritivas foram trocadas em períodos equidistantes de 7 dias.

As ementes da alface crespa, Cultivar $1=$ Elba, Cultivar $2=$ Cristina e Cultivar $3=$ Veneranda foram colocadas para germinar em bandejas e em espuma de fenólica com três cm de diâmetro e dois $\mathrm{cm}$ de altura previamente enxaguada com água corrente com o objetivo de eliminar possíveis compostos ácidos remanescentes de sua fabricação. Durante os primeiros seis dias as espumas foram umedecidas apenas com água de abastecimento da cidade de Campina Grande-PB; nos $7^{\circ}, 13^{\circ}$ e $19^{\circ}$ dias acrescentou-se a solução $\mathrm{S}_{1}$, de modo que ela apresentasse, respectivamente, 33,33; 66,66 e $100 \%$ da concentração nutricional sugerida por Furlani et al. (1995). As cultivares foram colocadas no perfil hidropônico definitivo após 24 dias da germinação.

A água utilizada para a formulação das soluções nutritivas foi proveniente da precipitação armazenada em cisterna e encaminhadas para análise físico-química no Laboratório de Irrigação e Salinidade (LIS/DEAg/UFCG) conforme os resultados (Tabela 3).

Tabela 3 - Caracterização físico-química das águas utilizadas nas irrigações do cultivo hidropônico.

\begin{tabular}{cc}
\hline Determinações & \\
\hline $\mathrm{pH}$ & 8,09 \\
Condutividade Elétrica $\left(\mathrm{dS} \cdot \mathrm{m}^{-1}\right)$ & 0,156 \\
Cálcio $\left(\mathrm{mmol}_{\mathcal{C}} / \mathrm{L}\right)$ & 1,20 \\
Magnésio $\left(\mathrm{mmol}_{\mathcal{C}} / \mathrm{L}\right)$ & 0,15 \\
Sódio $\left(\mathrm{mmol}_{\mathcal{C}} / \mathrm{L}\right)$ & 0,12 \\
Potássio $\left(\mathrm{mmol}_{\mathcal{C}} / \mathrm{L}\right)$ & 0,07 \\
Cloretos $\left(\mathrm{mmol}_{\mathcal{C}} / \mathrm{L}\right)$ & 0,50 \\
Carbonatos $\left(\mathrm{mmol}_{\mathcal{c}} / \mathrm{L}\right)$ & 0,20 \\
Bicarbonato $\left(\mathrm{mmol}_{\mathrm{c}} / \mathrm{L}\right)$ & 0,90 \\
Sulfatos & Ausente \\
Relação de adsorção de sódio $(\mathrm{RAS})$ & 0,15 \\
Classe de água para irrigação & $\mathrm{C} 1$ \\
\hline
\end{tabular}

Fonte: Dados da presente pesquisa.

Em função dos tratamentos as cultivares da alface foram avaliadas aos 22 dias após o transplantio para o perfil hidropônico definitivo quanto aos seguintes parâmetros: Clorofila total pelo uso do medidor portátil de SPAD-50, Clorofilas (Chl a, Chl b e Chl total) e carotenoides e Vitamina C.

A clorofila total foi determinada pelo uso do medidor portátil de clorofila SPAD-502 (Soil-Plant Analysis Development) Section. Minolta Camer Co., Ltd, Japão. 
Os teores de Chl $a$, Chl b e Chl total e carotenoides foram determinados pelo método descrito por Lichtenthaler (1987) e as análises foram quantificadas com Espectrofotômetro Digital UV-1000A, a absorbância da solução foi obtida por espectrofotometria nos comprimento de ondas de 470, 645 e $663 \mathrm{~nm}$.

Para a avaliação da Vitamina C, usou-se $15 \mathrm{~g}$ de amostra integral e preparação nos Erlenmeyers de $250 \mathrm{ml}$ contendo $50 \mathrm{ml}$ de ácido oxálico a 1\%. Soluções utilizadas: I - Solução de ácido oxálico 1\% (pesou-se $1 \mathrm{~g}$ de ácido oxálico PA e diluiuse em água deionizada até $100 \mathrm{~m} 1$ ); II - Solução de ácido ascórbico padrão $1 \mathrm{mg} \cdot \mathrm{m}^{-1}$ (pesou-se com precisão $0,05 \mathrm{~g}$ de ácido ascórbico padrão, estocado abrigado da luz. Transferiu-se para um balão volumétrico de $50 \mathrm{~m} 1$. Diluiu-se ao volume com solução de ácido oxálico 1\%. A solução foi preparada na hora do uso) e; III - Solução padrão de 2,6 diclorofenolindofenol (pesou-se $0,05 \mathrm{~g}$ de dicloroindofenol, que foi estocado em dessecador com soda e dissolveu-se com $50 \mathrm{ml}$ de água deionizada em balão volumétrico de $200 \mathrm{ml}$. Agitou-se vigorosamente e quando o corante dissolveu- se, diluiu-se a $200 \mathrm{ml}$ de água deionizada. Filtrou-se para um frasco de cor âmbar. Deixou-se estocado ao abrigo da luz e no refrigerador.

Os $15 \mathrm{~g}$ de amostra integral foram submetidos logo após a colheita à centrifugação em liquidificador, coou-se e transferiu-se três vezes $2,0 \mathrm{ml}$ da solução padrão de acido ascórbico para diferentes frascos de Erlenmeyers de $250 \mathrm{ml}$ contendo $50 \mathrm{ml}$ de ácido oxálico a $1 \%$. Titulou-se rapidamente com solução de indofenol através de bureta de $50 \mathrm{ml}$, até uma leve, mas distinta cor rósea persistente. Cada titulação consumiu entre 15 a $17 \mathrm{ml}$ de solução de indofenol. Similarmente titularam-se três brancos da mesma maneira usando água deionizada em lugar de solução de ácido ascórbico. Após diminuir da solução de indofenol gasta na titulação, a media da determinação dos brancos, calculou-se a concentração do indofenol como mg de ácido ascórbico equivalente a 1,0 ml de reagente. O calculo e a expressão dos resultados foi realizada segundo Brasil (1986).

Os dados obtidos foram submetidos à análise da variância pelo teste $\mathrm{F}$ a 1 e $5 \%$ de probabilidade. Quando verificado efeito significativo na análise da variância, as médias obtidas nas subparcelas (cultivares) foram comparadas pelo teste de Tukey e para parcelas (soluções) aplicou-se regressão. Os valores de Clorofila A e Caratenóides não seguiram as pressuposições do teste de normalidade (Shapiro-Wilk) para serem submetidos à ANOVA, portanto as médias foram comparadas pela estatística não paramétrica de Kruskal e Wallis. As análises estatísticas foram realizadas através do software estatístico SISVAR (Ferreira, 2014).

\section{Resultados e Discussão}

Analisando a Tabela 4, verifica-se que apenas o fator cultivar influenciou significativamente o teor de clorofila total analisado pelo índice indireto do SPAD a nível de $1 \%$ de probabilidade $(\mathrm{p}<0,01)$ nas cultivares $(\mathrm{C})$. 
Tabela 4 - Análise de variância da variável dos teores de clorofila total nas folhas pelo índice SPAD avaliada em campo no final do cultivo hidropônico das três cultivares da alface crespa submetidas aos diferentes tratamentos.

\begin{tabular}{|c|c|c|}
\hline FV & GL & QM \\
\hline & & SPAD \\
\hline Solução (S) & 6 & $0,09^{\mathrm{ns}}$ \\
\hline Bloco & 2 & $0,07^{\mathrm{ns}}$ \\
\hline erro 1 & 11 & 0,05 \\
\hline Cultivar (C) & 2 & $0,63^{\text {** }}$ \\
\hline $\mathrm{C} * \mathrm{~S}$ & 12 & $0,06^{\mathrm{ns}}$ \\
\hline erro 2 & 92 & 0,03 \\
\hline CV 1 & & 7,39 \\
\hline CV 2 & & 6,75 \\
\hline Média geral & & 2,95 \\
\hline
\end{tabular}

GL - grau de liberdade; FV - fonte de variação; QM - quadrado médio; ${ }^{\text {ns }}$ não significativo; ${ }^{* *}$ significativo a nível de $1 \%$ de probabilidade (p $<0,01$ ); pelo teste F; CV= coeficiente de variância. Parcelas e subparcelas, respectivamente.

Fonte: Dados da presente pesquisa.

Os teores de clorofila total nas folhas da alface pelo índice SPAD podem ser visualizados na Figura 2. O maior valor para clorofila total foi obtido na cultivar Veneranda cuja média correspondeu a 3,08 pelo índice SPAD.

Figura 2 - Determinação indireta dos teores de clorofila total nas folhas pelo índice SPAD em função do efeito isolado das cultivares de alface crespa Elba, Cristina e Veneranda ao final do experimento.

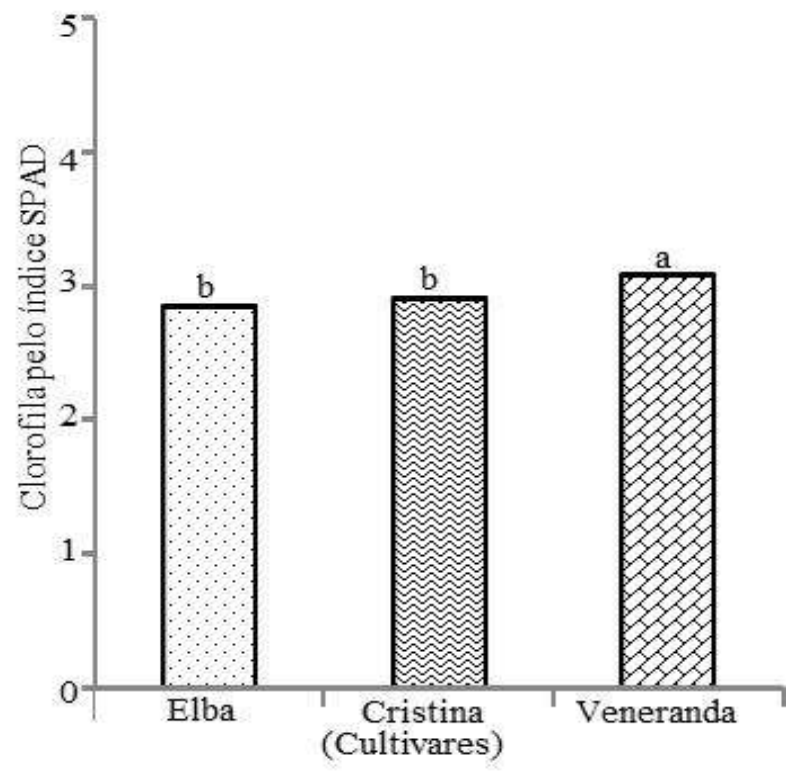

Fonte: Dados da presente pesquisa.

Santos et al. (2010) avaliaram o teor de clorofila na alface cultivada em sistema hidropônico, convencional e orgânico; e obtiveram os seguintes valores de 13,16; 12,72 e 13,22 respectivamente, estes valores também são distintos aos encontrados nesta pesquisa. Possivelmente o índice de clorofila encontrada neste estudo foi afetado pelos teores de cloreto de sódio ( $\mathrm{NaCl}$ ) adicionados nas soluções nutritivas. Estas oscilações de resultados evidenciam a importância de mais pesquisas sobre a interferência da salinidade sobre os diferentes tecidos vegetais. Analisando os dados da Figura 2, com os de Rosa et al. (2014) 
que estudaram a cultura da alface mimosa verde e roxa em sistema de cultivo hidropônico com condutividade elétrica da solução entre 1,85 e 2,00 dS/m $\mathrm{m}^{-1}$, os autores verificaram que a alface mimosa verde apresentou menores teores de clorofila.

Santos et al. (2010) avaliaram o teor de clorofila na alface cultivada em sistema hidropônico, convencional e orgânico; e obtiveram os seguintes valores de 13,16; 12,72 e 13,22 respectivamente, estes valores também são distintos aos encontrados nesta pesquisa. Possivelmente o índice de clorofila encontrada neste estudo foi afetado pelos teores de cloreto de sódio ( $\mathrm{NaCl}$ ) adicionados nas soluções nutritivas. Estas oscilações de resultados evidenciam a importância de mais pesquisas sobre a interferência da salinidade sobre os diferentes tecidos vegetais. Analisando os dados da Figura 2, com os de Rosa et al. (2014) que estudaram a cultura da alface mimosa verde e roxa em sistema de cultivo hidropônico com condutividade elétrica da solução entre 1,85 e 2,00 dS/ m$~^{-1}$, os autores verificaram que a alface mimosa verde apresentou menores teores de clorofila.

Verifica-se na Figura 3 o conteúdo dos teores de $C h l a$ em função das soluções nutritivas $S_{1}=1,9 ; S_{2}=2,4 ; S_{3}=2,9$; $\mathrm{S}_{4}=3,4 ; \mathrm{S}_{5}=3,9 ; \mathrm{S}_{6}=4,4$ e $\mathrm{S}_{7}=4,9 \mathrm{dS} \mathrm{m} \mathrm{m}^{-1}$ e das cultivares Elba, Cristina e Veneranda ao final do experimento, observa-se que as maiores médias encontradas foram nos tratamentos $C_{1} S_{2}\left(E l b a / S_{2}=2,4 \mathrm{dS} \mathrm{m}^{-1}\right)$ e $\mathrm{C}_{1} \mathrm{~S}_{3}\left(\mathrm{Elba} / \mathrm{S}_{3}=3,9 \mathrm{dS} \mathrm{m}^{-1}\right)$, apresentando os valores de 57 e $54 \mathrm{mg} / \mathrm{g}^{-1}$ na massa fresca da folha (MF). Comparando os teores de clorofila encontrados nesta pesquisa com os de Cardoso et al. (2018) que trabalharam com as cultivares dos grupos comerciais Lisa Repolhuda e Crespa Solta e observaram que o grupo Crespa Repolhuda apresentou teores de Chl a com média de 6,58 mg/g ${ }^{-1}$ este resultado é inferior aos desta pesquisa.

Figura 3 - Determinação dos teores de clorofila (Chl $\left.a \mathrm{mg} / \mathrm{g}^{-1} \mathrm{MF}\right)$ das cultivares de alface Elba, Cristina e Veneranda em função das soluções nutritivas $S_{1}=1,9 ; S_{2}=2,4 ; S_{3}=2,9 ; S_{4}=3,4 ; S_{5}=3,9 ; S_{6}=4,4$ e $S_{7}=4,9$ dS m${ }^{-1}$ ao final do experimento. Médias seguidas de mesma letra não diferem entre si.

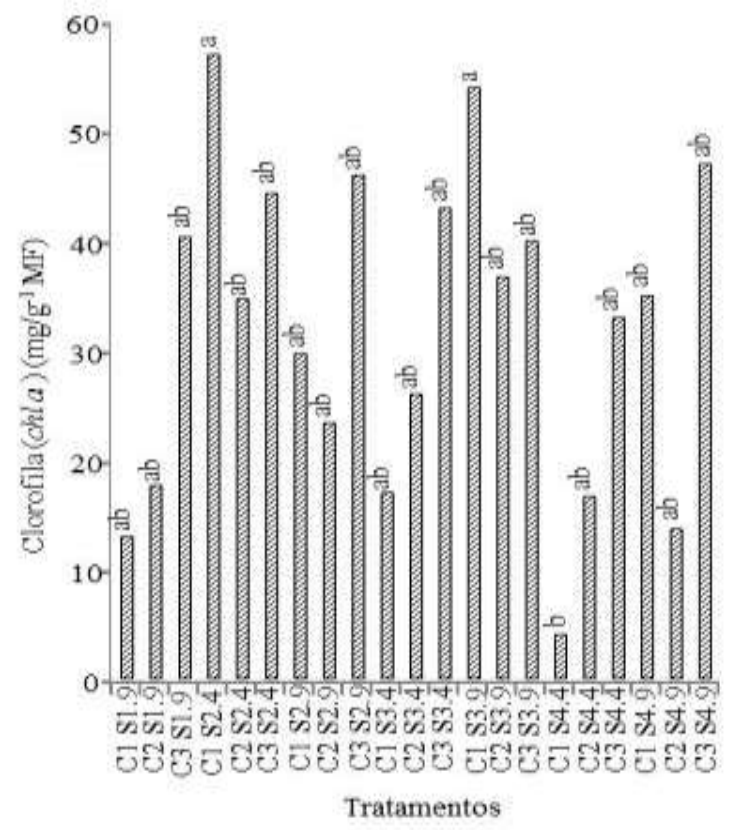

Fonte: Dados da presente pesquisa.

Observa-se na Tabela 5 que não houve efeito significativo para os teores cha b e cha total no fator solução nutritiva (S). Quanto aos resultados do efeito isolado no fator cultivar (C) os teores cha b e cha total apresentaram efeito significativo a nível de $1 \%$ de probabilidade ( $\mathrm{p}<0,01$ ). Para os resultados da interação da solução nutritiva dentro de cultivares e vice versa observa-se que não houve efeito significativo nos teores cha b e cha total (Tabela 5). 
Tabela 5 - Análise de variância dos teores de clorofila b e total na massa fresca (MF) das três cultivares da alface submetido aos diferentes tratamentos com soluções salinas.

\begin{tabular}{cccc}
\hline FV & GL & QM \\
\hline Soluções (S) & & Cha $\boldsymbol{b}$ & Cha total \\
Bloco & 6 & $0,54^{\mathrm{ns}}$ & $0,76^{\mathrm{ns}}$ \\
erro 1 & 2 & $0,25^{\mathrm{ns}}$ & $0,46^{\mathrm{ns}}$ \\
Cultivar (C) & 11 & 0,30 & 0,25 \\
C*S & 2 & $2,36^{* *}$ & $2,29^{* *}$ \\
erro 2 & 12 & $0,27^{\mathrm{ns}}$ & $0,34^{\mathrm{ns}}$ \\
CV 1 & 92 & 0,30 & 0,27 \\
CV 2 & & 17,07 & 13,79 \\
Média geral & & 17,2 & 14,39 \\
\end{tabular}

GL - grau de liberdade; ${ }^{\text {ns }}$ não significativo; ${ }^{* *}$ significativo a nível de $1 \%$ de probabilidade (p < 0,01); ${ }^{*}$ significativo a nível de $5 \%$ de probabilidade $(0,01 \leq \mathrm{p}<0,05)$ pelo teste $\mathrm{F} ; \mathrm{CV}=$ coeficiente de variância. Fonte: Dados da presente pesquisa.

Com relação teores de $C h l b$, para atender os pressupostos estatísticos da normalidade, os dados foram transformados em $\ln (\mathrm{x})$, sendo assim o comportamento observado na Figura 4, o conteúdo dos teores de Chl b em função das cultivares Elba, Cristina e Veneranda ao final do experimento, ainda na mesma figura observa-se que a maior média para $C h l b$ foi encontrada na cultivar Veneranda, cujo valor não transformado foi $\left(35,97 \mathrm{mg} / \mathrm{g}^{-1} \mathrm{MF}\right)$.

Figura 4 - Determinação dos teores de clorofila b $\left(C h l b \mathrm{mg} / \mathrm{g}^{-1} \mathrm{MF}\right)$ em função do efeito isolado das cultivares Elba, Cristina e Veneranda ao final do experimento. Médias seguidas de mesma letra não diferem entre si.

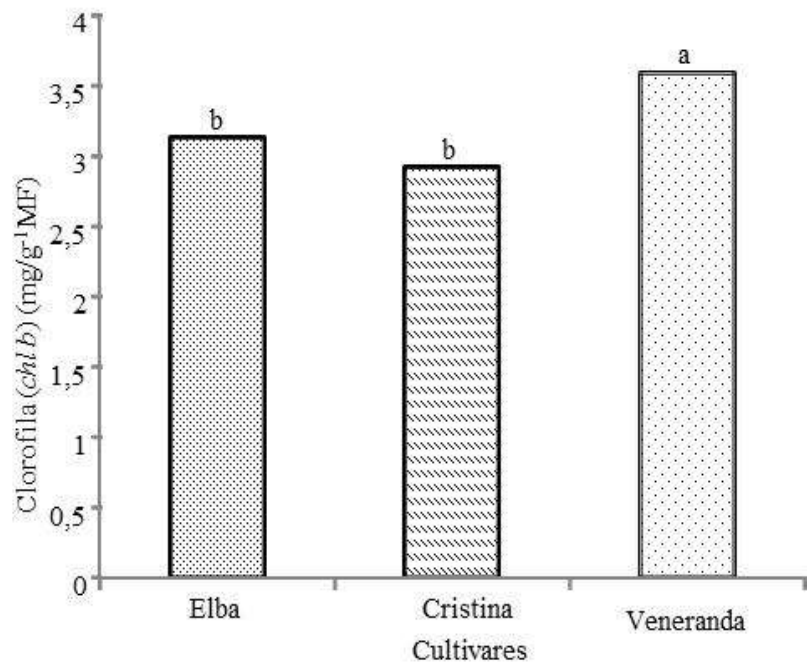

Fonte: Dados da presente pesquisa.

Comparando estes resultados com os de Xavier et al. (2019), que trabalhando em condições semelhante ao deste pesquisa obtiveram para o teor de $C h l b$ na cultivar Thais foram (17,54 mg/g-1 MF), esse valor é inferior aos desta pesquisa. Provavelmente esse valor inferior de $C h l b$ encontrados pelos autores foi devido à utilização de solução nutritiva preparada com água residuária do reator UASB (Upflow Anaerobic Sludge Blanket) que possivelmente quantidade de macro e micronutrientes existente nesta agua não favoreceu o desenvolvimento das plantas. Cardoso et al. (2018) analisaram cultivares 
dos grupos comerciais Lisa Repolhuda e Crespa Solta e observaram que o grupo Crespa Repolhuda apresentou teores $C h l b$ com média de $5,86 \mathrm{mg} / \mathrm{g}^{-1}$ esses resultados são distintos aos desta pesquisa.

Para atender as deduções estatísticas da normalidade, os dados de Chl total foram transformados em $\ln (\mathrm{x})$, sendo assim verifica-se na Figura 5 que maior teor médio de Chl total para a cultivar Veneranda $\left(52,06 \mathrm{mg} / \mathrm{g}^{-1} \mathrm{de} \mathrm{MF}\right)$. As cultivares Elba e Cristina apresentaram as seguintes médias de Chl total (35,04 e 27,07 mg/g-1 de MF) respectivamente e quando comparadas ao final do experimento, não diferiram estatisticamente entre si. Os valores de Chl total encontrado nesta pesquisa foram inferiores ao encontrado por Xavier et al. (2019), que verificou um teor de Chl total de $242 \mathrm{mg} / \mathrm{g}^{-1} \mathrm{MF}$ para cultivar Vanda utilizando a solução com água residuária domestica (esgoto bruto).

Figura 5 - Determinação dos teores de clorofila (Chl total $\mathrm{mg} / \mathrm{g}^{-1} \mathrm{MF}$ ) em função do efeito isolado das cultivares Elba, Cristina e Veneranda ao final do experimento. Médias seguidas de mesma letra não diferem entre si.

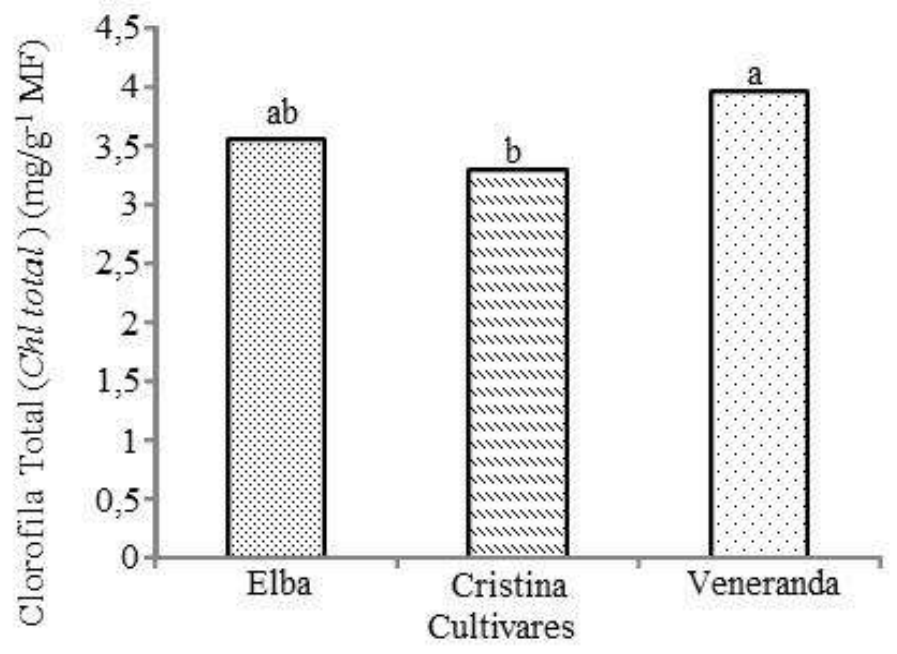

Fonte: Dados da presente pesquisa.

Observa-se na Figura 6 o conteúdo dos teores de carotenoides das cultivares Elba, Cristina e Veneranda em função das soluções nutritivas $\mathrm{S}_{1}=1,9 ; \mathrm{S}_{2}=2,4 ; \mathrm{S}_{3}=2,9 ; \mathrm{S}_{4}=3,4 ; \mathrm{S}_{5}=3,9 ; \mathrm{S}_{6}=4,4$ e $\mathrm{S}_{7}=4,9 \mathrm{dS} \mathrm{m}^{-1}$ ao final do experimento. Verifica-se que a maior média foi encontrada no tratamento $C_{1} S_{3}\left(\right.$ Elba/ $\left.\mathrm{S}_{3}=3,4 \mathrm{dS} \mathrm{m}^{-1}\right)$ cujo valor foi de $57 \mathrm{mg} / \mathrm{g}^{-1} \mathrm{MFF}$. Moura et al. (2016) trabalhando com alface roxo, fertilizante, sem pesticidas e com um composto de orgânico produzido a partir de esterco e resíduos de colheita da horta em período de desenvolvimento de 20, 30 e 40 dias após transplantio-DAT encontraram um alto conteúdo de carotenoides (128,098 $\left.\mathrm{mg} \mathrm{g}^{-1}\right)$ aos 40 DAT nas plantas. Silva et al. (2016) verificaram que houve incrementos nos teores de carotenoides à medida que houve aumento da aplicação de água nas plantas. 
Figura 6 - Determinação dos teores de carotenoides $\left(\mathrm{mg} / \mathrm{g}^{-1} \mathrm{MF}\right)$ nas folhas das cultivares da alface crespa em função das soluções nutritivas $\mathrm{S}_{1}=1,9 ; \mathrm{S}_{2}=2,4 ; \mathrm{S}_{3}=2,9 ; \mathrm{S}_{4}=3,4 ; \mathrm{S}_{5}=3,9 ; \mathrm{S}_{6}=4,4$ e $\mathrm{S}_{7}=4,9 \mathrm{dS} \mathrm{m}^{-1}$ e das cultivares $\mathrm{C}_{1}\left(\right.$ Elba), $\mathrm{C}_{2}$ (Cristina) e $\mathrm{C}_{3}$ (Veneranda) ao final do experimento. Médias seguidas de mesma letra não diferem entre si.

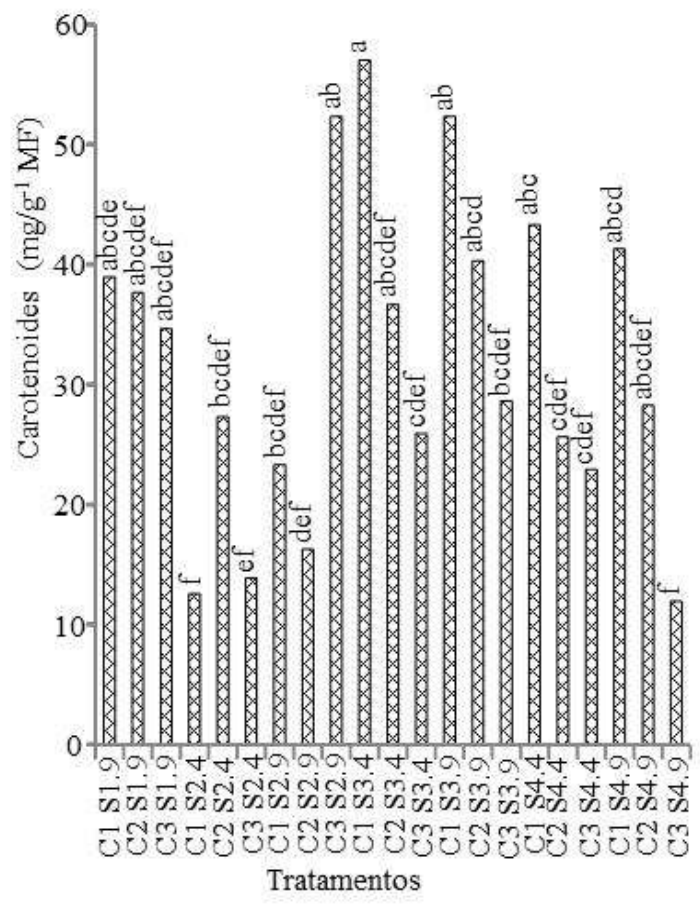

Fonte: Dados da presente pesquisa

A maioria das pesquisas sobre amadurecimento de vegetais é com base em avaliações na cor, sólidos solúveis totais e acidez (Motta et al., 2015), que sofrem mudanças consideráveis durante o desenvolvimento. Estes atributos básicos são essenciais para estabelecer o estágio de maturação, e importantes na detecção sensorial da qualidade do produto pelos consumidores (Carvalho et al., 2011; Ferreira et al., 2012). Outro atributo importante para a análise da qualidade da alface são carotenoides, que são substâncias que fornecem vários benefícios para a saúde humana.

Observa-se na Tabela 6 que o teor de vitamina C (TVC) no fator cultivar (C) apresentou efeito significativo a nível de $1 \%$ de probabilidade $(\mathrm{p}<0,01)$. Quanto aos resultados do efeito isolado da solução nutritiva (S) observa-se que os TVC apresentaram efeito significativo a nível de $5 \%$ probabilidade $(0,01 \leq p<0,05)$. Para os resultados da interação da solução nutritiva dentro de cultivares e vice versa observa-se que não houve efeito significativo nos TVC (Tabela 6). 
Tabela 6 - Análise de variância dos níveis de vitamina C (TVC) na massa fresca (MF) das três cultivares da alface submetido aos diferentes tratamentos com soluções salinas.

\begin{tabular}{ccc}
\hline FV & GL & QM \\
\hline Solução (S) & & Vitamina C- TVC mg.100g $\mathbf{~}^{\mathbf{1}}$ \\
Bloco & 6 & $9,42^{*}$ \\
erro 1 & 2 & 11,70 \\
Cultivar (C) & 11 & 4,49 \\
C*S & 2 & $121,23^{* *}$ \\
erro 2 & 12 & $8,68^{\text {ns }}$ \\
CV 1 & 92 & 4,15 \\
CV 2 & & 3,92 \\
Média geral & & 3,76 \\
\hline
\end{tabular}

GL - grau de liberdade; FV - fonte de variação; QM - quadrado médio; ${ }^{\text {ns }}$ não significativo; ${ }^{* *}$ significativo a nível de $1 \%$ de probabilidade $(\mathrm{p}<0,01)$; ${ }^{*}$ significativo a nível de $5 \%$ de probabilidade $(0,01 \leq \mathrm{p}<0,05)$ pelo teste $\mathrm{F}$; $\mathrm{CV}=$ coeficiente de variância.

Fonte: Dados da presente pesquisa.

Observa-se na Figura 7A que as soluções nutritivas apresentaram comportamento linear quanto aos TVC nas plantas de alface para todas as condutividades elétricas testadas; e não tiveram diferença estaticamente entre si. Quando se avalia as cultivares na Figura 7B verifica-se que as cultivares Cristina e Veneranda apresentarem os maiores teores de vitamina C, cujas médias foram 54,80 e $56,17 \mathrm{mg} .100 \mathrm{~g}^{-1}$, respectivamente.

Figura 7 - Determinação do teor de vitamina C (TVC mg.100 $\left.\mathrm{g}^{-1}\right)$ da alface crespa em função do efeito isolado das soluções nutritivas $S_{1} ; S_{2} ; S_{3} ; S_{4} ; S_{5} ; S_{6}$ e $S_{7}(A)$ e das cultivares Elba, Cristina e Veneranda (B). $S_{1}=100 \%$ da solução de Furlani preparada com água de chuva e $\left(\mathrm{S}_{2} ; \mathrm{S}_{3} ; \mathrm{S}_{4} ; \mathrm{S}_{5} ; \mathrm{S}_{6}\right.$ e $\left.\mathrm{S}_{7}\right)$ também preparadas utilizando água de chuva sendo adicionado cloreto de sódio $(\mathrm{NaCl})$ de modo a apresentarem as seguintes condutividades elétricas: $\left(\mathrm{S}_{2}=2,4 ; \mathrm{S}_{3}=2,9 ; \mathrm{S}_{4}=3,4 ; \mathrm{S}_{5}=3,9 ; \mathrm{S}_{6}=4,4\right.$ e $\left.\mathrm{S}_{7}=4,9 \mathrm{dS} \mathrm{m}^{-1}\right)$.
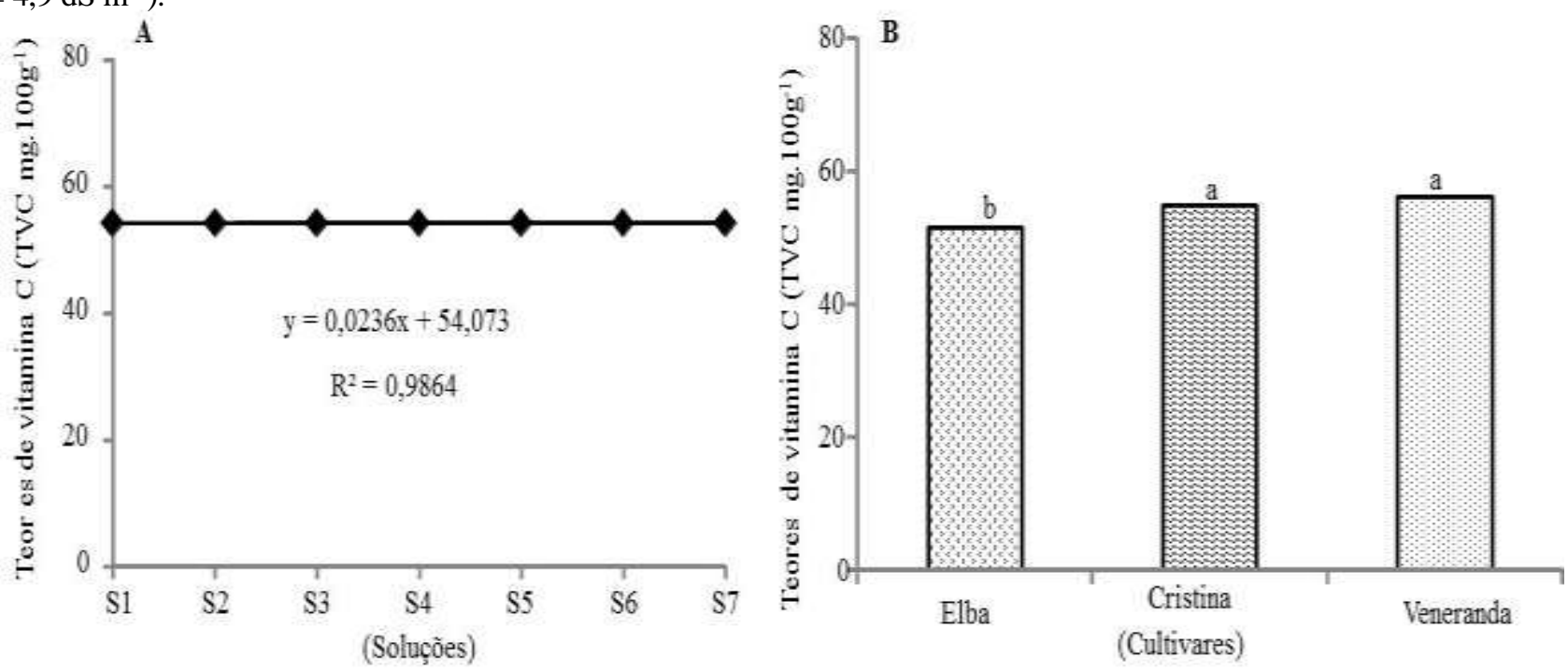

Fonte: Dados da presente pesquisa. 
Comparando os resultados encontrados nesta pesquisa verificou-se que os TVC foram superiores aos encontrados por Ohse et al. (2001), onde verificaram em sua pesquisa uma variação nos teores de vitamina C de 41,89 a 19,24 mg.100g-1. Correlacionando os resultados dos teores de vitamina $\mathrm{C}$ encontrados nesta pesquisa com os recomendados pela Tabela Brasileira de Composição dos Alimentos de 2011 (TBCA) verificou-se que os TVC encontrados no presente trabalho são superiores aos da TBCA, onde a mesma informa que em cada $100 \mathrm{~g}$ de alface fresca contem 15,6 mg de vitamina C.

Comparando os resultados dos teores de vitamina $\mathrm{C}\left(\mathrm{mg} .100 \mathrm{~g}^{-1}\right)$ encontrados nesta pesquisa, com os de Xavier et al. (2019), onde os mesmos trabalharam em condições semelhantes, com cultivares de alface e soluções nutritivas preparadas com água residuária e água salina de poço, os autores obtiveram teores de vitamina $\mathrm{C}\left(57,33 \mathrm{mg} .100 \mathrm{~g}^{-1}\right)$ para a cultivar Vanda, esse resultado corrobora com os encontrados nesta pesquisa. Gonçalves \& Coringa (2017) avaliaram o teor de vitamina C da alface coletada no comercio local de Cuiabá/MT cultivada em sistema hidropônico e encontraram teores de vitamina C (28,16 mg.100g $\mathrm{g}^{-1}$ ). Polette et al. (2018), encontraram valores de vitamina C para alface crespa crocante (SVR-2005) e Vanda (TE112) de $6,1 \mathrm{mg} .100 \mathrm{~g}^{-1}$ e $6,5 \mathrm{mg} .100 \mathrm{~g}^{-1}$ respectivamente, valores esses muito inferiores aos encontrados nessa pesquisa.

Sarmento et al. (2014) estudaram o teor de vitamina $C$ da alface e observaram o aumentou em resposta ao incremento

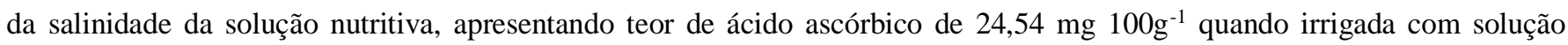

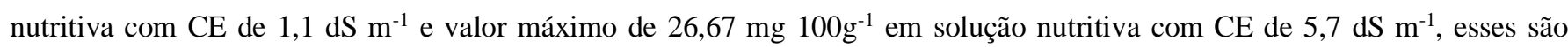
inferiores aos encontrados nesta pesquisa.

\section{Conclusão}

As soluções nutritivas apresentaram comportamento linear para todos os tratamentos e não diferiram estaticamente entre si.

A Clorofila (Chl a) obteve os maiores teores nos tratamentos $C_{1} S_{2}\left(E l b a / S_{2}=2,4 \mathrm{dS} \mathrm{m}^{-1}\right)$ e $C_{1} \mathrm{~S}_{3}\left(E l b a / S_{3}=3,9 \mathrm{dS} \mathrm{m}{ }^{-1}\right)$.

A cultivar Veneranda teve os maiores teores para a Clorofila (Chl b) e (Chl total).

As plantas do tratamento $\mathrm{C}_{1} \mathrm{~S}_{3}\left(\mathrm{Elba} / \mathrm{S}_{3}=3,4 \mathrm{dS} \mathrm{m} \mathrm{m}^{-1}\right)$ tiveram o maior conteúdo de carotenoides.

Os maiores teores de vitamina $\mathrm{C}$ foram encontrados nas cultivares Cristina e Veneranda.

O maior valor para clorofila total foi obtido para a cultivar Veneranda.

\section{Agradecimentos}

A Coordenação de Aperfeiçoamento de Pessoal de Nível Superior pelo apoio financeiro para realização da pesquisa.

Ao Centro de Ciências Agrárias e Ambientais-CCAA da Universidade Estadual da Paraíba-UEPB, Campus II, Lagoa Seca-PB por conceder a área para instalação do experimento e o Laboratório de Fitopatologia para realização das análises de Clorofila.

\section{Referências}

Alves, M. S., T. M; Luana T. Silva; Fernandes, J. P. Oliveira, M. L. A. \& Paz, V. P. S. (2011). Estratégias de uso de água salobra na produção de alface em hidroponia NFT. Revista Brasileira de Engenharia Agrícola e Ambiental. 15(1), 491-8. http://dx.org/10.1590/S1415-43662011000500009.

Ayers, R. S. \& Westcot, D. W. (1999). A qualidade de água na agricultura. (2a ed.), Universidade Federal da Paraíba- UFPB, FAO. 153. Campina GrandeParaíba.

Brasil, Ministério da Agricultura (1971). Levantamento exploratório-reconhecimento de solos do Estado da Paraíba. Equipe de Pedologia e Fertilidade do Solo. Divisão de agrologia - SUDENE. Boletim Técnico, 670.

Brasil. Leis, decretos, etc. Portaria n 076 de 27 de novembro de (1986). Diário Oficial da União, seção 1, 1986.18168.

Cardoso, S. S., Guimarães, M. de A., Lemos Neto, H. de S., Tello, J. P. de J. \& DoVale, J. C. (2018). Morphological and productive aspects of lettuce in low altitude and latitude. Revista Ciência Agronômica. 49(4), 644-62. http://dx.org/10.5935/1806-6690.20180073. 
Carvalho, A. V., Seccadio, L. L., Mourão Júnior, M.\& W. M. O. do Nascimento. (2011). Qualidade pós-colheita de cultivares de bananeira do grupo maçã, na região de Belém - PA. Revista Brasileira de Fruticultura, Jaboticabal. 33(4), 1095-102. http://dx.org/10.1590/S0100-29452011000400007.

Couto, M. A. L. \& Canniatti-Brazaca, S. G. (2010). Quantificação de vitamina C e capacidade antioxidante de variedades cítricas. Revista Ciência e Tecnologia de Alimentos. 30(1), 15-9. 10.1590/S0101-20612010000500003.

Dias, N. S., Jales, A. G. O., Sousa Neto, O. N., Gonzaga, M. I. S., Queiroz, I. S. R. \& Porto, M. A. F. (2011). Uso de rejeito da dessalinização na solução nutritiva da alface, cultivada em fibra de coco. Revista Ceres. 58,(1) 632-7. https://org/10.1590/S0034-737X2011000500014.

Ferreira, D. F. (2014). Estatística Básica. Editora Ufla, (2a ed.), 156. Ampliada e Revisada

Ferreira, R. M. A., Lopes, W. de A. R., Aroucha, E. M. M., Mano, N. C. S. \& Sousa, C. M. G. de. (2012). Caracterização física e química de híbridos de tomate em diferentes estádios de maturação produzidos em Baraúna, Rio Grande do Norte. Revista Ceres, Viçosa, 59(4), 506-11. http://dx.org/10.1590/S0034737 X2012000400011

Furlani, P. R. (1995). Cultivo de alface pela técnica de hidroponia - NFT. Campinas: IAC, Documentos, 55.18.

Gonçalves, T. O. \& Coringa, E. A. O. (2017). Caracterização físico-química e teor de nitrato em alface do tipo crespa e americana cultivadas sob sistema hidropônico e convencional. Higiene Alimentar. 31(1), 272-3.

Gondim, A. R. O., Flores, M. E. P., Martinez, H. E. P., Fontes, P. C. R. \& Pereira, P. R. G. (2010). Condutividade elétrica na produção e nutrição de alface em sistema de cultivo hidropônico NFT. Bioscience Journal. 26(1), 894-04. http://dx.org/10.18535/ijsrm.

Kluge, R. A., Tezotto-Uliana, J. V. \& Silva, P. P. M. (2015). Aspectos Fisiológicos e Ambientais da Fotossíntese. Revista Virtual de Química. 7(1), 56-73.

Lichtenthaler, H. K. (1987). Chlorophylls and carotenoids: pigments of photosynthetic biomembranes. In: Packer, L.; Douce, R. (Eds.). Methods in enzymology. Bad Honnef: Academic. 148(1), 350-82. http://dx.org/10.1016/0076-6879(87)48036-1.

Monteiro Filho, A. F., Azevedo, M. R. Q. A., Azevedo, C. A. V., Fernandes J. D., Silva, C. R. da \&. Silva, Y. dos S. (2017). Growth of hydroponic lettuce with optimized mineral and organomineral nutrient solutions. Revista Brasileira Engenharia Agrícola Ambiental. 21(1), 191-6. http://dx.org/10.1590/18071929.

Monteiro Filho, A. F., Pereira, G. L., Azevedo, M. R. Q. A., Fernandes J. D. \& Azevedo, C. A. V. (2014). Cultivo hidropônico de cultivares de alface em soluções nutritivas organominerais otimizadas com a ferramenta SOLVER. Revista Brasileira Engenharia Agrícola Ambiental. 18(4), 417-24. 10.1590/S141543662014000400009

Moura, L. O. Lanamar, C. A., Oliveira, K. G., Martins, L. M. \& Silva, E. C. (2016). Physicochemical characteristics of purple lettuce harvested at different ages. Revista Caatinga, Mossoró. 29(2), 489-95. http://dx.org/10.1590/1983-21252016v29n227rc.

Motta, J. D., Queiroz, A. J. de M., Figueirêdo, R. M. F. de. \& Sousa, K. dos S. M. de. (2015). Índice de cor e sua correlação com parâmetros físicos e físicoquímicos de goiaba, manga e mamão. Comunicata Scientiae, Bom Jesus. 6(1), 74-82. http://dx.org/10.14295/cs.v6i1.698.

Oliveira, L. L. P., Farias, W. C., Linhares, P. S. F., Melo, M. R. S., Cavalcante, J. J. \& Dombronski, J. L. D. (2014). Análise de diferentes dosagens de solução nutritiva no cultivo de mudas de alface americana (Lactuca sativa L.). Revista Agropecuária Científica no Semiárido. 10(1) 14-7. http://dx.org/10.30969/acsa.v10i2.503

Olshe, S., Dourado-Neto, D., Manfron, P. A. \& Santos, O. S. (2001). Qualidade de cultivares de alface produzidos em hidroponia. Scientia Agricola. 58(1), 181-85. http://dx.org/10.1590/S0103-90162001000100027.

Paulus, D., Dourado Neto, D., Frizzone, J. A. \& Soares, T. M. (2010). Produção e indicadores fisiológicos de alface sob hidroponia água salina. Revista Horticultura Brasileira. 28(1), 29-35. 10.1590/S0102-05362010000100006.

Polette, C. M. V., Sala, F. C., Spoto, M. H. F., Ferreira, M. D., Borba, K. R. \& Bernardi, M. R. V. (2018). Avaliação das características físico-químicas e aceitação da alface crocantela produzida em sistema hidropônico na cidade de Araras, São Paulo. Revista Demetra. 13, 663-673. https://org/: $10.12957 /$ demetra.2018.31509.

Rosa, A. M., Seó, H. L. S., Volpato, M. B., Foz, N. V., Silva, T. C., Oliveira, J. L. B., Pescador, R. \& Ogliari, J. B. (2014). Production and photosynthetic activity of Mimosa Verde and Mimosa Roxa lettuce in two farming systems. Revista Ceres. 61, 494-501. http://dx.org/10.1590/0034-737X201461040007.

Santos, R. S. S., Dias, N. S., Duarte, S. N. \& Lima, C. J. G. S. (2012). Uso de águas salobras na produção de rúcula cultivada em substrato de fibra de coco. Revista Caatinga. 25, 113-118. http://dx.org/10.1590/1983-21252018v31n424rc.

Santos, C. M. G., Braga, C. L., Vieira, M. R. S., Cerqueira, R. C. \& Brauer, R. L.; Lima, G. P. P. (2010). Qualidade da alface comercializada no município de Botucatu-SP. Revista Iberoamericana de Tecnologia Postcosecha. 10, 67-74.

Sarmento, J. D. A., Morais P. L. D., Almeida, M. B., Sousa Neto, O. N. \& Dias, N. S. (2014). Qualidade e Conservação da Alface Cultivada Com Rejeito Da dessalinização. Revista Caatinga. 27, 90-7. http://dx.org/10.13140/2.1.3532.3849.

Silva, W. D., Staboli, F. F., Costa, R., Rocha, L. C. D., Coletta, L. D. \& Anjos, M. P. (2018). Desenvolvimento de plantas de alface sob diferentes concentrações de biofertilizante. VI Congresso Latino-Americano, X Congresso Brasileiro, V Seminário do DF e Entorno. Brasília/DF. Cadernos de Agroecologia. 13(1).

Silva, A. R. A., Bezerra, F. M. L., Lacerda, C. F., Sousa, C. H. C. de. \& Chagas, K. L. (2016). Pigmentos fotossintéticos e potencial hídrico foliar em plantas jovens de coqueiro sob estresses hídrico e salino. Centro de Ciências Agrárias - Universidade Federal de Roraima, Boa Vista. Revista Agro@mbiente On-line. 10(4), 317-25. http://dx.org/10.18227/1982-8470ragro.v10i4.3650. 
Research, Society and Development, v. 10, n. 3, e10510313011, 2021

(CC BY 4.0) | ISSN 2525-3409 | DOI: http://dx.doi.org/10.33448/rsd-v10i3.13011

Silva, M. G., Soares, T. M., Oliveira, I. S., Santos, J. C. S., Pinho, J. S. \& Freitas, F. T. O. (2015). Produção de coentro em hidroponia NFT com o uso de águas salobras para reposição do consumo evapotranspirado. Revista Brasileira de Agricultura Irrigada. 9, 246-58. http://dx.org/10.7127/rbai.v9n400319.

Silva, A. O., Silva, D. J. R., Soares, T. M., Silva, E. F. F., Santos, A. N. \& Rolim, M. M. (2011). Produção de rúcula em sistema hidropônico NFT utilizando água salina do Semiárido-PE e rejeito de dessalinizador. Revista Brasileira de Ciências Agrárias. 6, 147-55. 10.5039/agraria.v6i1a929.

Souza Neta, M. L., Oliveira, F. A., Silva, R. T., Souza, A. A. T., Oliveira, M. K. T. \& Medeiros, J. F. (2013). Efeitos da salinidade sobre o desenvolvimento de rúcula cultivada em diferentes substratos hidropônicos. Revista Agro@mbiente. 7, 154-161. http://dx.org/10.18227/1982-8470ragro.v7i2.947.

Taiz, L.; Zeiger, E.; Moller, I. M. Murphy, A. (2017). Fisiologia e desenvolvimento vegetal. (6a ed.) Artmed, 858.

Taiz, L.; Zeiger, E. (2013). Fisiologia vegetal. (5a ed.), Artmed, 918.

Universidade Estadual de Campinas - UNICAMP. Tabela brasileira de composição de alimentos-TACO (2011). (4a ed.) rev. e ampl. Campinas: UNICAMP/NEPA. <http://www.unicamp.br/nepa/taco/tabela.php?ativo=tabela>.

Xavier, J. F., Azevedo, C. A. V., Azevedo, M. R. Q. A., Mendes, J. S., Fernandes J. D. \& Monteiro Filho A. F. (2019). Evaluation of vitamin C, nitrate and chlorophyll content determined in lettuce (Thaís, Vanda, Verônica) cultivated in hydroponic system using wastewater. Australian Journal of Crop ScienceAJCS - Austrália. 13(10): 1586-93. http://dx.org/10.21475/ajcs.19.13.06.p1682.

Xavier, J. F., Azevedo, C. A. V., Azevedo, M. R. Q. A., Lima, V. L. A., Dantas Neto, J. \& Santos, S. A. (2019). Determination of Microbiological Quality and Chlorophyll Levels Lettuce Grown Hydroponically With Wastewater. Journal of Agricultural Science, Cambridge. 11(1), 221-30. http://dx.org/10.5539/jas.v11n1p220. 